\title{
Magnetic Nanoparticles with Core Shell: Macroscopic Model and Coercive Field
}

\author{
O. Hudak ${ }^{1}$ and M. Hudak ${ }^{2}$ \\ ${ }^{1}$ Applied Physics Group, Department of Aerodynamics and Simulations, Faculty of Avionics, \\ Technical University of Kosice, Rampova 7, Stierova 23, SK-040 01 Kosice, Slovakia \\ ${ }^{2}$ Stierova 23, SK-040 23 Kosice, Slovakia \\ Correspondence should be addressed to O. Hudak, hudako@mail.pvt.sk \\ Received 4 March 2010; Accepted 3 June 2010 \\ Academic Editor: Steven L. Suib
}

Copyright (๑) 2010 O. Hudak and M. Hudak. This is an open access article distributed under the Creative Commons Attribution License, which permits unrestricted use, distribution, and reproduction in any medium, provided the original work is properly cited.

There exists nonhomogeneous structure in mechanosynthesized nanoparticles consisting of an ordered core surrounded by the shell. The shell may be structurally and magnetically disordered, or it may be ordered. These nanoparticles are found to be roughly spherical. We formulate the macroscopic model for the description of magnetic properties of nanoparticles with coreshell structure. The case of spheroids oriented in the same direction of polar axes is considered. There exits two coercive fields: $H_{c y}$ and $H_{c \psi}$. They are depending on quantities $o$ and $x$, where $x=V_{2} /\left(V_{1}-2 V_{2}\right)$, and $o=I_{F} / I_{S}$, here $V_{1}$ is the volume of the nanoparticle, and $V_{2}$ is the volume of the core. $I_{F}$ is the magnetization of the core, and $I_{S}$ is the magnetization of the shell. There exists a transition from one type of the coercive field to another, changing $x$ and $o$. We have found qualitative explanation of observed enhancement of coercive field and of its $d$-dependence in $\mathrm{NiFe}_{2} \mathrm{O}_{4}$ nanoparticles.

\section{Introduction}

Ferromagnetic and (ferri-) or antiferromagnetic nanoparticles, mechanochemically prepared, have a shell structure different from the core structure. The shell has mostly disordered structural and magnetic properties, while the core is magnetically ordered. In this paper, we formulate a model describing interaction of ellipsoid with ferromagnetic/antiferro- (ferri-)magnetic/ordering with shell (ellipsoid part which is not in the volume of the core ellipsoid). We study here the description of coercive fields of magnetic nanoparticles with spheroid shape with polar axes in the same direction for both spheroids. Due to fact that nanoparticles are small (smaller than $100 \mathrm{~A}^{\circ}$ ), we consider a single domain magnetic system. While porosity of ceramics prepared from such nanoparticles is in the range up to nearly 33 percents, and such pores do not disappear during further sintering, they undergo coalescence together with grain growth. Only subsurface pores have the possibility to go out in the surface, see in [1]. For the case of a spheroid with a pore, it can be easily shown that the pore may enhance the coercive field, or the coercive field is smaller than the bulk coercive field.

Mechanosynthesized $\mathrm{NiFe}_{2} \mathrm{O}_{4}$ nanocrystalline nanoparticles were studied in [2]. There exists nonhomogeneous structure in these nanoparticles consisting of an ordered core surrounded by a disordered grain boundary-surface region. The core has a fully inverse spinel structure with a collinear spin alignment. The surface layer (shell) is structurally and magnetically disordered due to nearly random distribution of cations and the canted spin structure. It exhibits a reduced nonsaturating magnetization, an enhanced coercivity, and a shifted hysteresis loop. The thickness of the shell of about $1 \mathrm{~nm}$ is estimated from size-dependent magnetization measurements. The shell thickness of crystallite radius ratio is nearly 23 percent. The nanoparticles are found to be roughly spherical with the core-shell structure. In [2], it was shown that the core part of the nanoparticle is structurally 
ordered and of nearly sphere shape, and the shell part is disordered structurally. The spins in the shell region are found to be canted $\left(28^{\circ}\right.$ and $40^{\circ}$ in magnetic sublattices), and the $\lambda$ parameter (parameter which in spinels describes how cations are distributed in spinels in their sublattices) equals nearly to 0.67 , in the core the $\lambda$ parameter is equal to 1 . It is observed that an enhanced magnetic hardness, that is, the coercive field of the material has the value $H_{C} \approx$ $0.35 \mathrm{~T}$ which is about 35 times larger than that for the bulk material $H_{C} \approx 0.01 \mathrm{~T}$. The field-cooled hysteresis loop is not symmetrical and is shifted to the left, and the shift is $\delta H_{C} \approx 0.03 \mathrm{~T}$. Such a shift and an asymmetric hysteresis loop were explained $[3,4]$ in the exchange model to be present due to the exchange coupling between the collinear spins in the core and canted spins in the shell. The saturation magnetization is dependent on the diameter. The growth of $\mathrm{NiFe}_{2} \mathrm{O}_{4}$ nanoparticle is accompanied by a decrease of coercivity field value.

The nonuniform core-shell structure of nanoparticles has been reported in $\mathrm{MgFe}_{2} \mathrm{O}_{4}$ [5] and in ball-milled nanocrystalline $\mathrm{LiNbO}_{3}$ [6]. Mechanochemically prepared nanoparticles $\mathrm{MgFe}_{2} \mathrm{O}_{4}$ [5] have an unusual property: enhanced magnetization. They have core-shell structure. Inner core exhibits a partly inverse structure with a Neel-type collinear spin alignment. The shell has a nonequilibrium cation distribution and a canted spin arrangement. Although spin canting effects tend to reduce the magnetic moment, the magnetization enhancement is attributed by authors to the nearly random distribution of magnetic cations in the shell region. On heating above $623^{\circ} \mathrm{K}$, the mechanochemical $\mathrm{MgFe}_{2} \mathrm{O}_{4}$ relaxes to the magnetic state which is similar to the bulk one [5]. The thickness $t$ of the shell assuming the spherical shape is estimated to be $t \approx 1 \mathrm{~nm}$ and is comparable to the lattice constant.

Nanocomposite $\mathrm{CuFe}_{2} \mathrm{O}_{4} / \mathrm{CeO}_{2}$ [7] has a ferrimagnetic behaviour. The nanoparticle size of $\mathrm{CuFe}_{2} \mathrm{O}_{4} / \mathrm{CeO}_{2}$ decreases with increasing concentration of $\mathrm{CeO}_{2}$. Added $\mathrm{CeO}_{2}$ is dissolved in the $\mathrm{CuFe}_{2} \mathrm{O}_{4}$, or it is coated on the surface of $\mathrm{CuFe}_{2} \mathrm{O}_{4}$ nanoparticles [8]. There is then a surface layer-shell of $\mathrm{CeO}_{2}$. This form of core (shell) structure is of different type, as in $\mathrm{NiFe}_{2} \mathrm{O}_{4}$. Nevertheless, also in this case there is a core and a shell, and they have magnetic properties [9]. It is possible to model such materials as materials in which there is a core-shell structure similar to that in nanoparticles prepared by mechanochemical preparation. Modelling of $\mathrm{CeO}_{2-x}$ in the $\mathrm{LDA}+\mathrm{U}$ formalism has been used to account for the strong on-site Coulomb repulsion among the localized Ce $4 \mathrm{f}$ electrons. Results show that by choosing an appropriate $\mathrm{U}$, it is possible to consistently describe structural, thermodynamic, and electronic properties o $\mathrm{CeO}_{2-x} \mathrm{f}$, which enables modelling of redox processes involving ceria-based materials. We may expect that similar nanocomposites may be prepared using $\mathrm{CeAl}_{2}$ as a shell. We have modelled $\mathrm{CeAl}_{2}$ nanoparticles [10] by $1 \mathrm{~d}$ Hubbard model. Surface valence phase transition between magnetic and nonmagnetic states was studied by us [10] in these nanoparticles. Coated $\mathrm{CeAl}_{2}$ may form the coreshell structure. The surface properties of the shell may be described by our model [10] (surface quantum phase transition) and also by the model of magnetic nanoparticles with core-shell in which the coercive field is calculated here.

There is different surface anisotropy for the cubic and spherical nanoparticles [11]. Low temperature properties: saturation magnetization, coercivity, and spin canting are similar, while the blocking temperature $T_{B}$ (temperature at which a maximum of susceptibility in nanoparticle is achieved) is different for $\gamma-\mathrm{Fe}_{2} \mathrm{O}_{3}$ for the cubic and spherical shape. Theoretical calculations and experimental magnetic studies for nanoparticles of different shapes are [12-17] scare, see in [11]. For cobalt ferrite $\mathrm{CoFe}_{2} \mathrm{O}_{4}$, it was found that blocking temperature $T_{B}$ and the saturation magnetization are independent of the shape, and the value of the coercivity field $H_{C}$ is larger for spherical shape than for the cubic one. In cobalt ferrite there is a larger magnetic anisotropy. For $\gamma-\mathrm{Fe}_{2} \mathrm{O}_{3}$, intrinsic magnetocrystalline anisotropy is small. Monte Carlo simulations are based on Heisenberg model for exchange interaction between spins in the core and in the shell experiencing different anisotropies [11]. The surface thickness is taken to be equal to one lattice constant. Experimentally it was found that the spherical nanoparticles are more ordered than cubic nanoparticles. Low temperature measurements show however that their microscopical magnetic structure properties are similar, indicating similar crystalline properties. The behaviour is ascribed to the effects of morphology on the effective surface anisotropy and the small value of intrinsic anisotropy of $\gamma-\mathrm{Fe}_{2} \mathrm{O}_{3}$. The magnetic state in the core is ferrimagnetic and in the surface layer is of the spin-glass type. We will take into account the morphology effects in our paper by considering the ellipsoidal shape of nanoparticles. This shape enables us to describe the spherical shape of nanoparticles, the disc-like shape, or rod-like shape for extremal values of lengths axes in the ellipsoid. The case of cubic nanoparticles can be easily considered in the same way.

In the next section, we formulate the core-shell model for description of magnetic nanoparticles. In the third section, the Model Free Energy is found. In the next sections we study the free energy for spheroids and spheres oriented in the same direction. Experimental behaviour of coercive field dependence on diameter of nanoparticle is compared with theoretical predictions. We have found that there exist two coercive fields $H_{c \gamma}$ and $H_{c \psi}$. They are depending on quantities $o$ and $x$, defined by $x=V_{2} /\left(V_{1}-2 V_{2}\right)$, and by $o=I_{F} / I_{S}$, here $V_{1}$ is the volume of the larger ellipsoid, and $V_{2}$ is the volume of the smaller ellipsoid. $I_{F}$ is the magnetization of the volume where smaller ellipsoid occurs, and $I_{S}$ is the magnetization of the shell. There exists a transition from one type of the coercive field to another changing $x$ and $o$ within range of their possible values. Critical values $x_{c}$ and $o_{c}$ for which values of both coercive fields are equal can be found. For spheroidal shapes of ellipsoids for $x>x_{c}$, the field $H_{c y}$ is the coercive field which may be observed in experiments. For volumes $V_{1}$ and $V_{2}$ for which $x<x_{c}$, the field $H_{c \psi}$ is the coercive field and should be observed. The ratio of the coercive field value for nanoparticle to the bulk coercive field value is different for $x>x_{c}$ and for $x<x_{c}$. Enhancement 


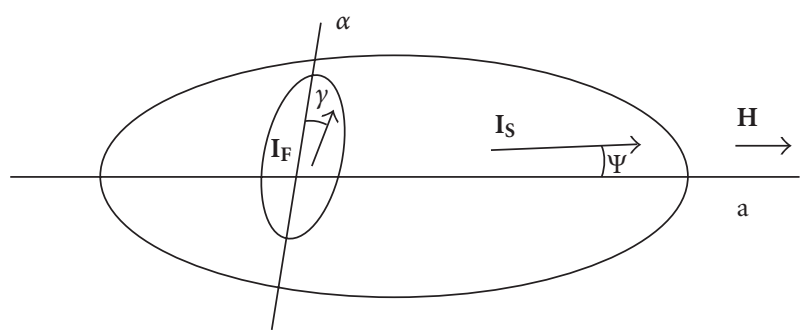

FIgURE 1: Core-shell magnetic structure. The larger ellipsoid has a homogeneous magnetization $\mathbf{I}_{\mathbf{S}}$, and the smaller ellipsoid has a homogeneous magnetization $\mathbf{I}_{\mathbf{F}}-\mathbf{I}_{\mathbf{S}}$. There is a total magnetization $\mathbf{I}_{\mathbf{F}}-\mathbf{I}_{\mathbf{S}}+\mathbf{I}_{\mathbf{S}}=\mathbf{I}_{\mathrm{F}}$ in the volume where smaller ellipsoid (core) is present in the larger one. The angle of the magnetization of the shell volume with the direction of the main axis is $\psi$, and the angle of the magnetization of the core with the direction of the main axis is $\gamma$.

of the coercive field may occur in nanoparticles changing the diameter $d$.

\section{Core-Shell Model}

As it was shown above, there are many nanoparticle systems in which there exists core-shell structure. They are found in nanoparticles prepared, for example, by mechanochemical method and also by other methods. For an overview of the mechanochemical methods see in [18].

We will describe such nanoparticles by the following Core-Shell model. Due to small diameter of nanoparticles we will assume that they are magnetically monodomain nanoparticles. This is true for nanoparticles with diameter less than $100 \mathrm{~A}^{\circ}$ [19]. The shape of nanoparticles described above is of the sphere type, mostly, there are deviations from this shape in the core and in the shell. To describe these observations, we assume that the nanoparticle has an ellipsoid shape in general. The ellipsoid shape is described by three parameters $a, b$, and $c$ (lengths of axes). To describe existence of the core and of the shell, we consider another ellipsoidal "nanoparticle" which is located inside the first one. The centre of that another "nanoparticle" may have almost any position inside the first ellipsoid, assuming that the second ellipsoid is as a whole inside the first ones volume.

We will assume that main axes in both ellipsoids coincide with the polar (light magnetic) crystallographic axis of the nanoparticle. In general, these two axes (light and main axes in both ellipsoids) are oriented in different directions. We will denote the angle between polar axis in both by $\theta$. The case of zero angle, $\theta=0$, corresponds to parallel polar axes. The case of angle $\theta=\pi / 2$ corresponds to perpendicular polar axes. Here in this paper we consider the case of $\theta=0$ angle which corresponds to the shell and the oriented core in the same direction.

To describe core-shell magnetic structure we will assume that the larger ellipsoid has a homogeneous magnetization $\mathbf{I}_{\mathrm{S}}$, and that the smaller ellipsoid has a homogeneous magnetization $\mathbf{I}_{\mathbf{F}}-\mathbf{I}_{\mathbf{S}}$. Thus, there is a total magnetization $\mathbf{I}_{\mathbf{F}}-$ $\mathbf{I}_{\mathbf{S}}+\mathbf{I}_{\mathbf{S}}=\mathbf{I}_{\mathbf{F}}$ in the volume where smaller ellipsoid is localized in the larger one, see Figure 1. The magnetic structure of the material in core and in shell may be ferromagnetic or ferri- (antiferro-) magnetic. We will assume that sublattice magnetizations are coupled strongly, and that then we may consider magnetizations $\mathbf{I}_{\mathbf{F}}$ and $\mathbf{I}_{\mathbf{S}}$ (resulting magnetization) only. We will neglect also surface magnetic phenomena. The magnetic field around an ellipsoid of the sphere shape with homogeneous magnetization $\mathbf{I}_{\mathbf{F}}-\mathbf{I}_{\mathbf{S}}$ is given in our case by the dependence [19]

$$
\left(I_{F}-I_{S}\right) \frac{4 \pi}{3} \frac{(d / 2)^{3}}{r^{3}}\left(2 \mathbf{e}_{r} \cos (\theta)+\mathbf{e}_{\rho} \sin (\theta)\right)
$$

where $r$ is the distance from the centre of the sphere, and $d$ is a diameter of the sphere. Here, $\mathbf{e}_{r}$ is a unit vector in the direction of radius directing from the centre, and $\mathbf{e}_{\rho}$ is a unit vector in the direction perpendicular to the vector $\mathbf{e}_{r}$. For larger distances from the smaller ellipsoid surface, we may neglect this contribution. This approximation corresponds to smaller inner ellipsoid volume with respect to the larger ones volume.

Let us consider first the case in which both ellipsoids are spheres. Then we will consider the case of spheroids (ellipsoids with the same two main axes lengths). The angle of the magnetization of the first (larger) ellipsoid with the direction of the main axis is $\psi$, and the angle of the magnetization of the second (smaller) ellipsoid with the direction of the main axis is $\gamma$, see Figure 1.

\section{Model-Free Energy}

Interaction of the core magnetization with the shell magnetization will be described by the effective-field method. We will assume that an effective magnetic field of the larger ellipsoid is acting on the smaller ellipsoid magnetization. On the other hand, the effective magnetic field of the smaller ellipsoid outside and inside this ellipsoid which is acting on the larger ellipsoid magnetization will be neglected.

The magnetic anisotropy of the shell structure may be described by the unit vector $\mathbf{e}_{\mathbf{S}}$, and the contribution to the free energy is of the form $K_{S}\left(\mathbf{e}_{\mathbf{S}} \cdot \mathbf{I}_{\mathbf{S}}\right)^{2}$, where $K_{S}$ is the anisotropy constant for the shell. The anisotropy of the core may be described by the unit vector $\mathbf{e}_{\mathrm{C}}$, and the contribution to the free energy is of the form $K_{C}\left(\mathbf{e}_{\mathbf{C}} \cdot \mathbf{I}_{\mathrm{F}}\right)^{2}$, where $K_{C}$ is the magnetic anisotropy constant for the core. When the structure is random then the anisotropy is random, and we can use mean anisotropy of the magnetic structure in the shell. Random anisotropy in the shell (for the constant $\lambda$ unequal to one) may be described by random orientation of the vector $\mathbf{e}$ and by the different values of the constant $K$. We will neglect magnetic anisotropy in this paper as the magnetic anisotropy constants $K_{S}$ and $K_{C}$ are small in $\mathrm{NiFe}_{2} \mathrm{O}_{4}$.

We will assume that the nanoparticle is monodomain, and the free energy of the magnetic system will contain contributions similar to those for the ellipsoid with an ellipsoidal hollow [19]. The inner ellipsoid is corresponding to the hollow in [19], however we consider this ellipsoid as a magnetic system with the magnetization $\mathbf{I}_{\mathrm{F}}-\mathbf{I}_{S}$ then the 
total magnetization in the volume $V_{2}$ of smaller ellipsoid is $\mathbf{I}_{\mathbf{F}}-\mathbf{I}_{\mathbf{S}}+\mathbf{I}_{\mathbf{S}}=\mathbf{I}_{\mathrm{F}}$. The volume of the larger ellipsoid is $V_{1}$.

Let us denote susceptibilities for larger and smaller ellipsoids as $\chi_{S}^{0}$ and $\chi_{F}^{0}$ respectively, they are defined as

$$
\chi_{S}^{0}=\frac{1}{N_{a} t_{\mathrm{a}}^{2}+N_{b} t_{b}^{2}+N_{c} t_{c}^{2}},
$$

where $N_{i}$ is a demagnetization factor for the direction $i(i=$ $a, b, c)$ for the larger ellipsoid, and where $t_{i}$ is a directional cosine of the magnetization vector $\mathbf{I}_{\mathbf{S}}$ for the direction $i$, and

$$
\chi_{F}^{0}=\frac{1}{N_{\alpha} t_{\alpha}^{2}+N_{\beta} t_{\beta}^{2}+N_{\gamma} t_{\gamma}^{2}},
$$

where $N_{j}$ is a demagnetization factor for the direction $j(j=$ $\alpha, \beta, \epsilon)$ for the smaller ellipsoid, and where $t_{j}$ is a directional cosine of the magnetization vector $\mathbf{I}_{\mathbf{S}}$ for the direction $j$.

The free energy $F_{S}$ of the larger ellipsoid is

$$
F_{S}=\frac{1}{2} V_{1} \frac{1}{\chi_{S}^{0}} \mathbf{I}^{2} .
$$

The free energy $F_{F}$ of the smaller ellipsoid is

$$
F_{F}=\frac{1}{2} V_{2} \frac{1}{\chi_{F}^{0}}\left(\mathbf{I}_{\mathbf{F}}-\mathbf{I}_{\mathbf{S}}\right)^{2} .
$$

The free energy $F_{S F}$ describing the interaction of the effective magnetic field - - $\left(1 / \chi_{S}^{0}\right) \mathbf{I}_{S}$ of the larger ellipsoid magnetization with the magnetization $\mathbf{I}_{\mathrm{F}}-\mathbf{I}_{\mathrm{S}}$ of the smaller ellipsoid within the volume $V_{2}$ of the smaller ellipsoid is

$$
F_{S F}=\frac{1}{2} V_{2} \frac{1}{\chi_{F}^{0}} \mathbf{I}_{\mathbf{S}} \cdot\left(\mathbf{I}_{\mathbf{F}}-\mathbf{I}_{\mathbf{S}}\right) .
$$

The free energy describing the interaction of the magnetic field $\mathbf{H}$ with the magnetization $\mathbf{I}_{\mathbf{S}}$ of the larger ellipsoid within the volume $V_{1}$ is

$$
F_{H 1}=-V_{1} \mathbf{I}_{\mathbf{S}} \cdot \mathbf{H} \text {. }
$$

and the free energy describing the interaction of the magnetic field $\mathbf{H}$ with the magnetization $\mathbf{I}_{\mathbf{F}}-\mathbf{I}_{\mathbf{S}}$ within the volume of "second nanoparticle" $V_{2}$ is

$$
F_{H 2}=-V_{2}\left(\mathbf{I}_{\mathbf{F}}-\mathbf{I}_{\mathbf{S}}\right) \cdot \mathbf{H} .
$$

Let us introduce the spherical coordinates for magnetizations $\mathbf{I}_{\mathbf{S}}$ and $\mathbf{I}_{\mathbf{F}}$ :

$$
\mathbf{I}_{\mathbf{S}}=I_{S}(l, m, n)
$$

where $I_{S}$ is the amplitude of the magnetization $\mathbf{I}_{S}$ of the larger ellipsoid, and $(l, m, n)$ are coordinates of the directional unit vector of magnetization $\mathbf{I}_{\mathbf{S}}$, and

$$
\mathbf{I}_{\mathrm{F}}=I_{F}(L, M, N),
$$

where $I_{F}$ is the amplitude of the magnetization of the larger ellipsoid, and $(L, M, N)$ are coordinates of the unit vector of the direction of magnetization $\mathbf{I}_{\mathbf{F}}$. Note that $l^{2}+m^{2}+n^{2}=1$ and that $L^{2}+M^{2}+N^{2}=1$.

\section{Free Energy: Spheroids Oriented in the Same Direction}

Let us consider the case of spheroids with polar axes in the same direction. This case corresponds to the cases of mechanochemically prepared nanoparticles in which the shell has the direction of polar axis the same as the direction of polar axis of the core spheroid and $\theta=0$. The free energy for this case has the form $F=F_{S}+F_{F}+F_{S F}+F_{H 1}+F_{H 2}$.

The total free energy $F$ for the case of spheroids with the polar axis in the same direction has the form

$$
\begin{aligned}
F= & F_{0}+\frac{1}{2} I_{S}^{2}\left(N_{a}-N_{b}\right)\left(V_{1}-2 V_{2}\right) \cos ^{2}(\psi) \\
& +\frac{1}{2} I_{F}^{2}\left(N_{a}-N_{b}\right) V_{2} \cos ^{2}(\gamma) \\
& -V_{2} I_{S} I_{F} \cos (\gamma+\psi)\left(N_{a}-N_{b}\right)\left(\cos ^{2}(\gamma)-\cos ^{2}(\psi)\right) \\
& -\left(V_{1}-V_{2}\right) I_{S} H \cos (\psi)-V_{2} I_{F} H \cos (\gamma),
\end{aligned}
$$

where $F_{0}$ is the free energy which does not depend on angles $\gamma$ and $\psi$. We assume that the deviation of magnetization vectors $\mathbf{I}_{S}$ and $\mathbf{I}_{\mathrm{F}}$ from polar axes in core and in shell is small, thus angles $\psi$ and $\gamma$ are small angles with values near 0 .

The free energy constant $F_{0}$ has the form

$$
F_{0}=\frac{1}{2} V_{1} N_{b} I_{S}^{2}+\frac{1}{2} V_{2} N_{b} I_{S}^{2}\left(I_{F}^{2}+I_{S}^{2}\right)-V_{2} N_{b} I_{S}^{2} .
$$

\section{The Case of Spheres}

Let us now discuss simple case of two spheres. Note that centres of these spheres are not necessary in the same point. In this case we have $N_{a}-N_{b}=0$ and $N_{\alpha}-N_{\beta}=0$. Thus in the case of the spheres there are only last two terms present in (11). The total free energy $F$ for the case of spheroids with the polar axis in the same direction is

$$
F=F_{0}-\left(V_{1}-V_{2}\right) I_{S} H \cos (\psi)-V_{2} I_{F} H \cos (\gamma),
$$

where $F_{0}$ is the free energy which does not depend on the angles $\gamma$ and $\psi$ (see above).

Note that for $H>0$, the lowest free energy (13) corresponds to angles $\psi=0$ and $\gamma=0$. For $H<0$, the lowest free energy (13) corresponds to angles $\psi=\pi$ and $\gamma=\pi$. Thus spins in both spheres are oriented in the direction of the magnetic field. The magnetization $M$, its component in the direction of main axis, has in this simple case the form:

$$
M=\left(V_{1}-V_{2}\right) I_{S} \cos (\psi)+V_{2} I_{F} \cos (\gamma) .
$$

Thus the difference of magnetization for $H>0$ and for $H<0$ has the form for the angles $\psi$ and $\gamma$ which minimizes free energy:

$$
\Delta M=2\left(V_{1}-V_{2}\right) I_{S}+2 V_{2} I_{F} .
$$

The coercive field has the zero value $H_{c, \pm}=0$. The magnetic susceptibility $\chi$, has in this case value

$$
\chi=\frac{\delta M}{\delta H}=2 \Delta M \delta(H) .
$$


In fact the delta function above will be a peak like (a gaussian peak) with a finite and with an amplitude given by $2\left(V_{1}-V_{2}\right) I_{S}+2 V_{2} I_{F}$

\section{The Case of Spheroids}

Let us now discuss the case of spheroids with the same shape, in which $N_{a}-N_{b}$ is different from 0 and $N_{\alpha}-N_{\beta}$ is different from $0, N_{a}-N_{b}=N_{\alpha}-N_{\beta}$. Assuming small angles $\psi$ and $\gamma$ we can find the following form of the free energy expansion in $\psi$ and $\gamma$ :

$$
\begin{aligned}
F= & F_{1}+I_{S}^{2}\left(N_{a}-N_{b}\right)\left(V_{1}-2 V_{2}\right) \\
\times\left[\psi^{2}\left(-\frac{1}{2}-x \cdot o+H \frac{1+x}{2 I_{S}\left(N_{a}-N_{b}\right)}\right)\right. & \\
& \left.\quad+\gamma^{2}\left(-x \frac{1+o^{2}}{2}+H x o \frac{1}{2 I_{S}\left(N_{a}-N_{b}\right)}\right)\right],
\end{aligned}
$$

where $F_{1}$ is a constant not dependent on angles $\gamma$ and $\psi$. As we can see from (17) using the method from [19], there are two coercive fields, which we denote as $H_{c \gamma}$ and $H_{c \psi}$, at which coefficients of $\psi^{2}$ and $\gamma^{2}$ become zero:

$$
H_{c \gamma}=I_{S}\left(N_{a}-N_{b}\right) \frac{\left(1+o^{2}\right)}{o}
$$

$($ for $x \neq 0)$ and

$$
H_{c \psi}=I_{S}\left(N_{a}-N_{b}\right) \frac{1+2 x o}{1+x} .
$$

Here the quantities $o$ and $x$ are defined by $x=V_{2} /\left(V_{1}-\right.$ $\left.2 V_{2}\right)$ and $o=I_{F} / I_{S}$. The coercive field which will be measured in experiments is that field from coercive fields $H_{c \gamma}$ and $H_{c \psi}$, which is smaller and in this case both magnetizations $\mathbf{I}_{\mathbf{S}}$ and $\mathrm{I}_{\mathrm{F}}$ are reoriented. When this field is approached decreasing the magnetic field from above, there will be a change in the sign of the coefficient corresponding free energy terms. Note that for small values of $x$ for which the core is small, this field becomes $H_{c \psi}=I_{S}\left(N_{a}-N_{b}\right)(1+x(2 o-1))$ and thus is increasing with increasing $x$ for $2 o>1$, is larger than the bulk coercive field. For $2 o<1$ it is smaller than the bulk coercive field

From (18) and (19) we can find conditions for critical values of $x$ and $o$ quantities for which values of both coercive fields $H_{c \gamma}$ and $H_{c \psi}$ are equal:

$$
\frac{1+2 x o}{1+x}=\frac{\left(1+o^{2}\right)}{o} .
$$

The coercive fields $H_{c \psi}$ and $H_{c y}$ change their role as the observed coercive field at those values of $o$ and $x$ for which (20) holds.

The critical value $x_{c}$ at which this change takes place is given by:

$$
x_{c}=\frac{1-o+o^{2}}{o^{2}-1} .
$$

We see that at $o=0$ where there is zero magnetization in the core, we have the critical value of $x$ such that $V_{1}=V_{2}$. At $o=\infty$ we have the critical value of $x$ such that $V_{2}=V_{1} / 3$. There is zero magnetization $I_{S}=0$ in the shell now. For $o=$ $1_{+}$, we find $x_{c}=+\infty$ and $V_{2}=V_{1} / 2$, for $o=1_{-}$we obtain $x_{c}=-\infty$ and again $V_{2}=V_{1} / 2$. There is the same magnetic moment in both core and shell for $o=1_{+}$and $o=1_{-}$.

For the same spheroidal shapes of ellipsoids for $x>x_{c}$ the field $H_{c y}$ is the coercive field observed in experiments. For volumes $V_{1}$ and $V_{2}$ for which $x<x_{c}$, the field $H_{c \psi}$ is the coercive field observed, now the smaller spheroid is very small. The bulk value of the coercive field as can be easily shown is given by

$$
H_{c b \psi}=I_{S}\left(N_{a}-N_{b}\right) .
$$

\section{Discussion}

The coercive field depends on the diameter $d$ in nanoparticles prepared by mechanochemical methods. The nanoparticle growth of $\mathrm{NiFe}_{2} \mathrm{O}_{4}$ is accompanied by the decrease of coercivity [2]. The surface to volume fraction in this material decreases with increasing annealing temperature. In [20], it was found that increasing the diameter $d$ of the nanoparticle the width of the shell decreases for $\mathrm{BaFe}_{12} \mathrm{O}_{19}$ and for $\mathrm{Co}_{0.56} \mathrm{Zn}_{1.44} \mathrm{~W}$. While this fact was found to be true for nanoparticles with the diameter $d$ from the interval $100 \mathrm{~A}^{\circ}$ to $400 \mathrm{~A}^{\circ}$ we will assume that the same qualitative dependence of thickness $t$ on the diameter $d$ is present for diameter $d<$ $100 \mathrm{~A}^{\circ}$ in $\mathrm{NiFe}_{2} \mathrm{O}_{4}$ nanoparticles. From two coercive fields, which we have found above, the field $H_{c \psi}$ depends on the volume of nanoparticles: $H_{c \psi}=H_{c b \psi}((1+2 x o) /(1+x))$. Here $H_{c b \psi}$ is a bulk value of the coercive field, $H_{c b \psi}=I_{S}\left(N_{a}-N_{b}\right)$. This coercive field changes with the diameter $d$ as

$$
\frac{d}{d d} H_{c \psi}=H_{c b} \frac{2 o-1}{(1+x)^{2}} \frac{d x}{d d} .
$$

As we can see the change of the coercive field $H_{c \psi}$ on the diameter $d$ is given by $d x / d d$. This change is found to be

$$
\frac{d x}{d d}=\frac{V_{1} V_{2}^{\prime}-V_{2} V_{1}^{\prime}}{\left(V_{1}-2 V_{2}\right)^{2}}
$$

Here $V_{1}^{\prime}=d V_{1} / d d$ and $V_{2}^{\prime}=d V_{2} / d d$. We can find that the coercive field $H_{c \psi}$ is decreasing for $o>1 / 2$ with increasing diameter $d$ when the condition $t(d)>d(d t(d) / d d)$, where $t(d)$ is $d$-dependent width of the shell. $d$-dependent width of the shell is decreasing with increasing diameter $d$ as is observed in some experiments [20], in [2], it is assumed that the thickness of the shell in $\mathrm{NiFe}_{2} \mathrm{O}_{4}$ does not depend on $d$. In both cases the condition $t(d)>d(d t(d) / d d)$ is fulfilled. While the spin $I_{S}$ of the shell is canted from the spin $I_{F}$ of the core by angles $20^{\circ}$ and $40^{\circ}$ depending on the sublattice, the value of the spin in the shell may be expected to be smaller than the value of the spin in the core due to disorder in the shell and the condition $o>1 / 2$ may be assumed to be satisfied. Thus our theory qualitatively explains observed decrease of the coercive field with increasing diameter $d$ of nanoparticles for nanoparticles with the width of the shell independent of diameter or with decreasing width of the shell with increasing diameter $d$. In the shell of 
nanocrystalline complex oxides a nonequilibrium cation distribution was found accompanied by a deformation of polyhedron geometries [21]. Note for example that in some nanoparticles like BPFTO $\left(\left(\mathrm{Bi}_{0.9} \mathrm{~Pb}_{0.1}\right)\left(\mathrm{Fe}_{0.9} \mathrm{Ti}_{0.1}\right) \mathrm{O}_{3}\right)$, the width of the shell with increasing diameter may increase. For small increase of the shell width which authors are describing as almost constant, see [22].

The coercive field observed in experiment in $\mathrm{NiFe}_{2} \mathrm{O}_{4}$ is enhanced by the factor 35 with respect to the coercive field of the bulk [2]. The ratio of the coercive field $H_{c \psi}$ to the bulk coercive field $H_{c b \psi}$ is given as $\left(1+2 x_{0}\right) /(1+x)$. We then obtain that the value of $o$ corresponding to the enhancement factor in $\mathrm{NiFe}_{2} \mathrm{O}_{4}$ is $o=35+17 / x$ and we see that for small volumes $V_{2}$ of the core the ratio o of spin values is too large. The spins in the shell do not have large value. For larger values of the volume $V_{2}$ and larger values of shell spins the enhancement factor may be expected to have the value 35 . In our approximation of a small $x$ the enhancement of the coercive field may exist, however this enhancement does not reach the value 35; one can find $(d / d x)\left(\left(1+2 x_{0}\right) /(1+x)\right)=$ $2(2 o-1) /(1+x)^{2}>0$ for $o>1 / 2$ and for $x \approx 0$. We have $(1+2 x o) /(1+x) \approx 1+x(2 o-1)>1$, however this ratio does not reach the value 35 in $\mathrm{NiFe}_{2} \mathrm{O}_{4}$.

\section{Acknowledgments}

The author would like to express his thanks to Professor V. Sepelak for discussions on nanoparticles prepared mechanochemically. This paper was supported by the Slovak Research and Development Agency under the contract no. APVV-0728-07.

\section{References}

[1] V. V. Zyryanov, V. A. Sadykov, M. I. Ivanovskaya, J. M. Criado, and S. Neophytides, "Synthesis and sintering of ceramic nanocomposites with high mixed conductivity," Science of Sintering, vol. 37, no. 1, pp. 45-54, 2005.

[2] V. Šepelák, I. Bergmann, A. Feldhoff et al., "Nanocrystalline nickel ferrite, $\mathrm{NiFe}_{2} \mathrm{O}_{4}$ : mechanosynthesis, nonequilibrium cation distribution, canted spin arrangement, and magnetic behavior," Journal of Physical Chemistry C, vol. 111, no. 13, pp. 5026-5033, 2007.

[3] R. H. Kodama, A. E. Berkowitz, E. J. McNiff Jr., and S. Foner, "Surface spin disorder in ferrite nanoparticles (invited)," Journal of Applied Physics, vol. 81, no. 8, pp. 5552-5557, 1997.

[4] R. H. Kodama and A. E. Berkowitz, "Atomic-scale magnetic modeling of oxide nanoparticles," Physical Review B, vol. 59, no. 9, pp. 6321-6336, 1999.

[5] V. Šepelák, A. Feldhoff, P. Heitjans et al., "Nonequilibrium cation distribution, canted spin arrangement, and enhanced magnetization in nanosized $\mathrm{MgFe}_{2} \mathrm{O}_{4}$ prepared by a one-step mechanochemical route," Chemistry of Materials, vol. 18, no. 13, pp. 3057-3067, 2006.

[6] P. Heitjans, M. Masoud, A. Feldhoff, and M. Wilkening, "NMR and impedance studies of nanocrystalline and amorphous ion conductors: iithium niobate as a model system," Faraday Discussions, vol. 134, pp. 67-82, 2007.

[7] R. K. Selvan, C. O. Augustin, V. Šepelák, L. J. Berchmans, C. Sanjeeviraja, and A. Gedanken, "Synthesis and characterization of $\mathrm{CuFe}_{2} \mathrm{O}_{4} / \mathrm{CeO}_{2}$ nanocomposites," Materials Chemistry and Physics, vol. 112, no. 2, pp. 373-380, 2008.

[8] Z. Zhang, Z. Gong, and Y. Yang, "Electrochemical performance and surface properties of bare and $\mathrm{TiO}_{2}$-coated cathode materials in lithium-ion batteries," Journal of Physical Chemistry B, vol. 108, no. 45, pp. 17546-17552, 2004.

[9] D. A. Andersson, S. I. Simak, B. Johansson, I. A. Abrikosov, and N. V. Skorodumova, "Modeling of $\mathrm{CeO}_{2}, \mathrm{Ce}_{2} \mathrm{O}_{3}$, and $\mathrm{CeO}_{2-\mathrm{x}}$ in the $L D A+U$ formalism," Physical Review B, vol. 75, no. 3, Article ID 035109, 6 pages, 2007.

[10] O. Hudak, "Quantum critical phenomena, entanglement entropy and Hubbard model in $1 \mathrm{~d}$ with the boundary site with a negative chemical potential $-p$ and the Hubbard coupling $U$ positive," Physics Letters, Section A, vol. 373, no. 3, pp. 359$362,2009$.

[11] G. Salazar-Alvarez, J. Qin, V. Šepelák et al., "Cubic versus spherical magnetic nanoparticles: the role of surface anisotropy," Journal of the American Chemical Society, vol. 130, no. 40, pp. 13234-13239, 2008.

[12] Q. Song and Z. J. Zhang, "Shape control and associated magnetic properties of spinel cobalt ferrite nanocrystals," Journal of the American Chemical Society, vol. 126, no. 19, pp. 6164-6168, 2004.

[13] W. Seo, H. Jo, K. Lee, B. Kim, S. Oh, and J. Park, "Sizedependent magnetic properties of colloidal $\mathrm{Mn}_{3} \mathrm{O}_{4}$ and $\mathrm{MnO}$ nanoparticles," Angewandte Chemie International Edition, vol. 116, no. 9, pp. 1135-1137, 2004.

[14] D. A. Dimitrov and G. M. Wysin, "Effects of surface anisotropy on hysteresis in fine magnetic particles," Physical Review B, vol. 50, no. 5, pp. 3077-3084, 1994.

[15] R. Evans, U. Nowak, F. Dorfbauer et al., "The influence of shape and structure on the Curie temperature of $\mathrm{Fe}$ and $\mathrm{Co}$ nanoparticles," Journal of Applied Physics, vol. 99, no. 8, Article ID 08G703, 3 pages, 2006.

[16] R. Yanes, O. Chubykalo-Fesenko, H. Kachkachi, D. A. Garanin, R. Evans, and R. W. Chantrell, "Effective anisotropies and energy barriers of magnetic nanoparticles with Néel surface anisotropy," Physical Review B, vol. 76, no. 6, Article ID 064416, 13 pages, 2007.

[17] J. Bai, Y.-H. Xu, and J.-P. Wang, "Cubic and spherical highmoment FeCo nanoparticles with narrow size distribution," IEEE Transactions on Magnetics, vol. 43, no. 7, pp. 3340-3342, 2007.

[18] V. V. Zyryanov, "Mechanochemical synthesis of complex oxides," Russian Chemical Reviews, vol. 77, no. 2, pp. 105-135, 2008.

[19] V. I. Ivanovskij and L. A. Cernikova, Fizika Magnitnych Javlenij-Seminari, Izd, Moskovskogo Univerziteta, Moscow, Russia, 1981.

[20] E. P. Naiden, R. A. Zhuravlyov, V. I. Itin et al., "Magnetic properties of hexaferrite nanosized powders produced via mechanoactivation," Science of Sintering, vol. 37, no. 2, pp. 107-114, 2005.

[21] V. Šepelák, I. Bergmann, A. Diekmann, P. Heitjans, and K. D. Becker, "Mechanosynthesis of nanocrystalline iron germanate $\mathrm{Fe} 2 \mathrm{GeO} 4$ with a nonequilibrium cation distribution," Reviews on Advanced Materials Science, vol. 18, no. 4, pp. 349-352, 2008.

[22] K. Singh, R. K. Kotnala, and M. Singh, "Study of electric and magnetic properties of $\left(\mathrm{Bi}_{0.9} \mathrm{~Pb}_{0.1}\right)\left(\mathrm{Fe}_{0.9} \mathrm{Ti}_{0.1}\right) \mathrm{O}_{3}$ nanomultiferroic system," Applied Physics Letters, vol. 93, no. 21, Article ID 212902, 3 pages, 2008. 

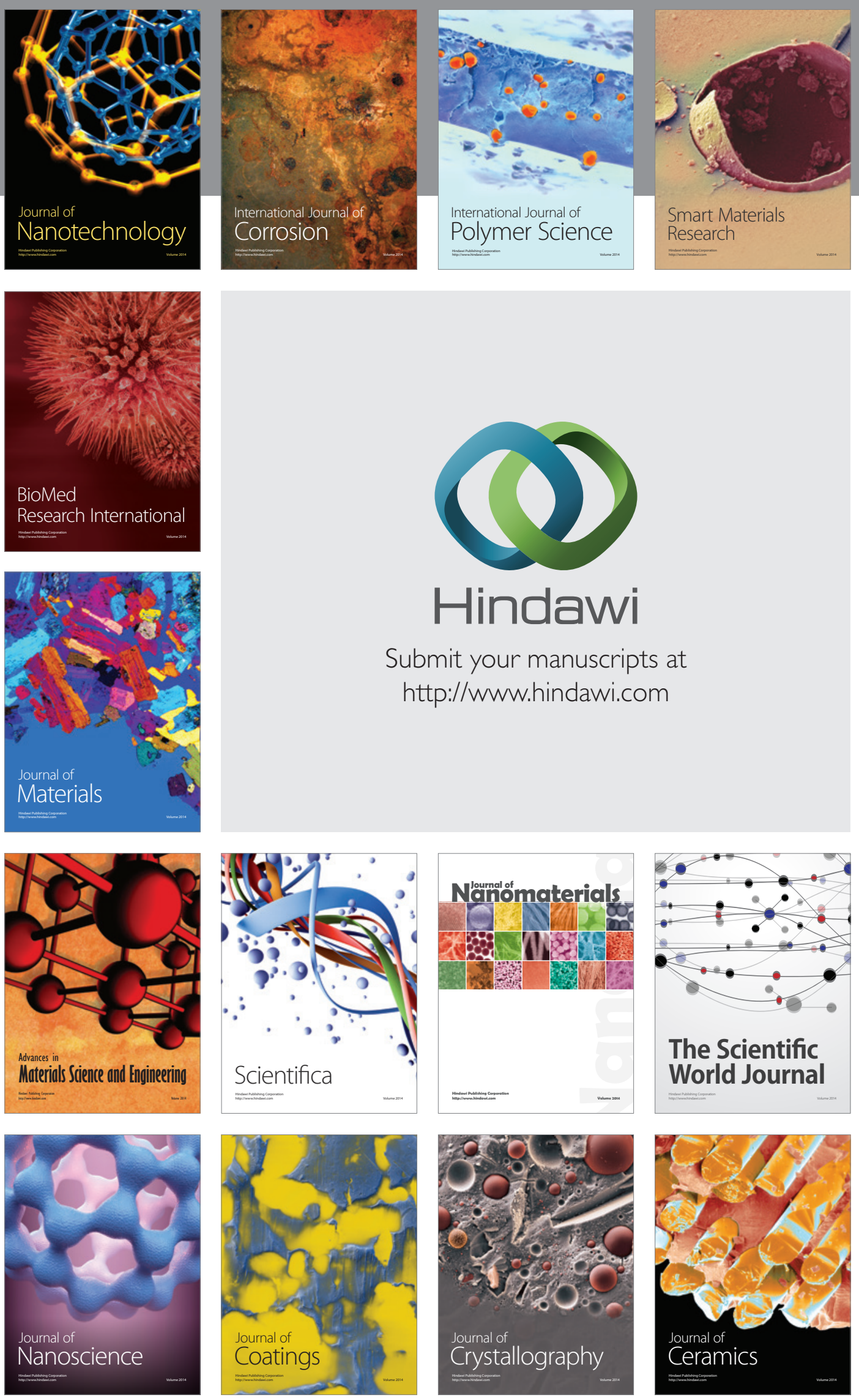

The Scientific World Journal

Submit your manuscripts at

http://www.hindawi.com

\section{World Journal}

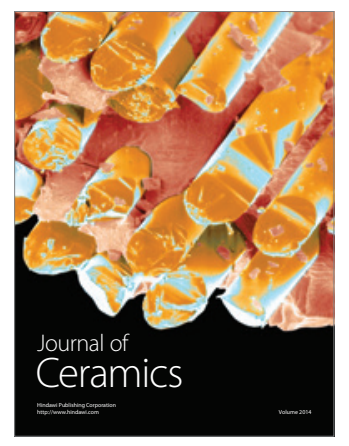

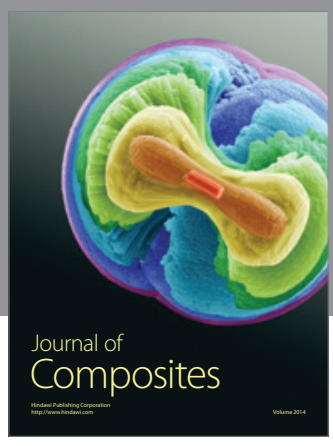
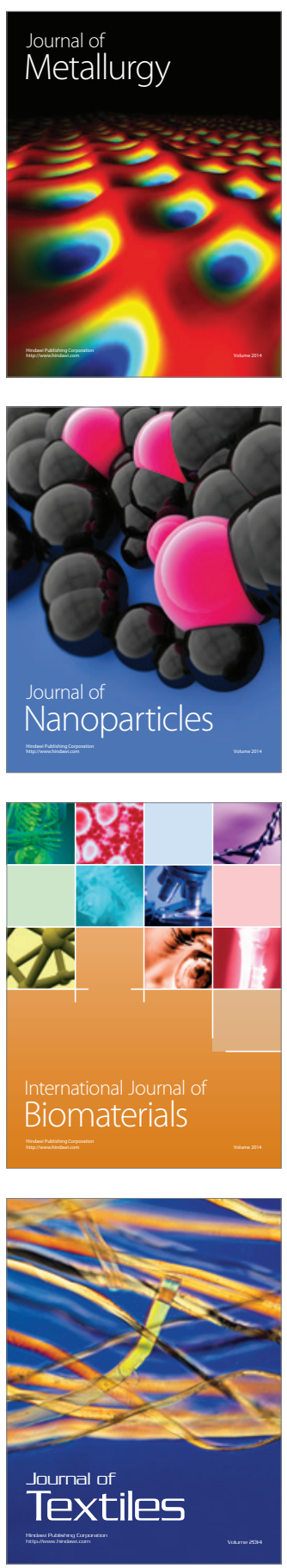